\title{
Assessment of the Impacts of Livestock Grazing on Endangered Red Panda (Ailurus fulgens) Habitat in Eastern Nepal
}

\author{
Sony Lama ${ }^{1,2^{*}}$, Saroj Shrestha ${ }^{1^{*}}$, Narayan Prasad Koju ${ }^{2,3,4^{*}}$, Ang Phuri Sherpa ${ }^{1}$, \\ Munmun Tamang 1 \\ ${ }^{1}$ Red Panda Network, Kathmandu, Nepal \\ ${ }^{2}$ Naaya Aayam Multi-disciplinary Institute (NAMI College), Jorpati, Kathmandu, Nepal \\ ${ }^{3}$ Center for Post Graduate Studies (nec-CPS), Nepal Engineering College, Bhaktapur, Nepal \\ ${ }^{4}$ Department of Psychology, University of Washington, Seattle, Washington, USA \\ Email: *sony.lama@redpandanetwork.org, ${ }^{*}$ saroj.shrestha@redpandanetwork.org, ${ }^{\star}$ narayan.koju@nami.edu.np
}

How to cite this paper: Lama, S., Shrestha, S., Koju, N.P., Sherpa, A.P. and Tamang, M. (2020) Assessment of the Impacts of Livestock Grazing on Endangered Red Panda (Ailurus fulgens) Habitat in Eastern Nepal. Open Journal of Ecology, 10, 97-110. https://doi.org/10.4236/oje.2020.103008

Received: December 9, 2019

Accepted: March 20, 2020

Published: March 23, 2020

Copyright $\odot 2020$ by author(s) and Scientific Research Publishing Inc. This work is licensed under the Creative Commons Attribution International License (CC BY 4.0).

http://creativecommons.org/licenses/by/4.0/

\begin{abstract}
Disturbances caused by livestock grazing are considered as a key attributable factor for the habitat loss of red panda. The present study was carried out in Eastern Nepal mainly focusing on the habitat preferences of red panda and impacts of livestock grazing on it. In total, 15 linear transects were delineated at an elevation interval of $100 \mathrm{~m}$ assessing indirect signs of red panda and livestock. Habitat preferences of red panda, vegetation abundance, coverage percentage of vegetation layers (canopy and shrub layer) and distance to water source were recorded using randomly plotted 58 quadrats of $10 \mathrm{~m} \times 10 \mathrm{~m}$. Questionnaire survey and Key Informant Interviews were carried out to evaluate the perception of local community on conservation threats and importance of red panda conservation. Jaccard's similarity index was used to determine the spatial habitat overlap of livestock and red panda. Litsea elongata, Lithocarpus pachyphylla and Rhododendron falconeri were the most common species in the study area. Grazing signs were observed from $51 \%$ of red panda habitats in the study area. Livestock herding has induced negative impacts on the habitat of red panda inside the community forests. The study findings will extend the existing body of knowledge regarding the status of red panda in Eastern Himalayas. In addition, this research will help in the formulation of effective legal and strategic framework of sustainable grazing management.
\end{abstract}

\section{Keywords}

Habitat Preference, Jaccard's Similarity Index, Habitat Overlap, Red Panda, Livestock Grazing 


\section{Introduction}

Recognizing environmental and anthropogenic factors which affect distribution and survival of species is a key pursuit of ecology [1] [2]. Information related to habitat characteristics and potential threats is very crucial in formulating an effective conservation-dependent species' management strategy [3] [4]. Red panda (Endangered-IUCN Red List, Appendix I-CITES), inhabits eastern Himalayan temperate broadleaf forests with bamboo in the understory within a preferred altitudinal range of 2400 - $3900 \mathrm{~m}$ [5] [6]. Red panda is a flagship species of the Eastern Himalayan eco-region with its distribution limited in five countries: China, Myanmar, India, Bhutan and Nepal [5] [7]. It prefers forests dominated by Betula utilis, Rhododendron spp, Abies spp and bamboo species in the understory [8]. Glaston et al. [9] have estimated the global population of red panda to be around 10,000 individuals. The report on Population and Habitat Viability Assessment (PHVA) estimated the total population of red panda ranging from 237 to 1061 individuals in Nepal [10]. However, adequate information about the habitat preferences, ecology and conservation threats to red panda is limited [11] [12].

Although red panda is protected by national laws in all the range countries, their numbers in the wild continue to decline due to habitat loss, fragmentation and poaching [13] [14] [15]. Traditional transhumance grazing method is the major cause of habitat loss and fragmentation in Nepal [6]. Livestock grazing causes abrupt diminution of understory/bamboo species [16]. Increase in the livestock grazing activities has negative impacts on the populations of red panda [17] [18]. In central Nepal, grazing has deteriorated red panda habitat and declined its population [6]. Similarly, Sharma et al. [19] recorded the adverse effects of livestock presence in red panda habitat in Western Nepal. However, Bartolome et al. [20] and Barry et al. [21] suggested that regulated livestock grazing can be an alternative conservation tool in managing the grasslands from invasive plant species and minimizing catastrophic wildland fires.

The current study determines the effects of livestock grazing on red panda habitat suitability in Eastern Nepal and provides some robust information on the habitat preferences of this species. The study aims to offer a scientific basis to promote the endorsement of effective conservation strategies necessary for prolonged conservation of such endangered species.

\section{Study Area}

This study was conducted in two community forests of Eastern Nepal: Singhadevi Community forest and Chipchipe Community forest, which lies in the critical Panchthar-Ilam-Taplejung (PIT) Corridor (Figure 1). The study site falls under Kangchenjunga Singhalila Complex (KSC) which provides connectivity between protected areas in Nepal and India [22]. Singhadevi CF $\left(26^{\circ} 59^{\prime} 37.5^{\prime \prime} \mathrm{N}\right.$ and $88^{\circ} 4^{\prime} 56.06^{\prime \prime} \mathrm{E}$ ) extends over 231 hectares of land whereas Chipchipe CF $\left(26^{\circ} 59^{\prime} 41.28^{\prime \prime} \mathrm{N}\right.$ and $\left.88^{\circ} 4^{\prime} 54.08^{\prime \prime} \mathrm{E}\right)$ covers 430 hectares of land. The altitude of the 


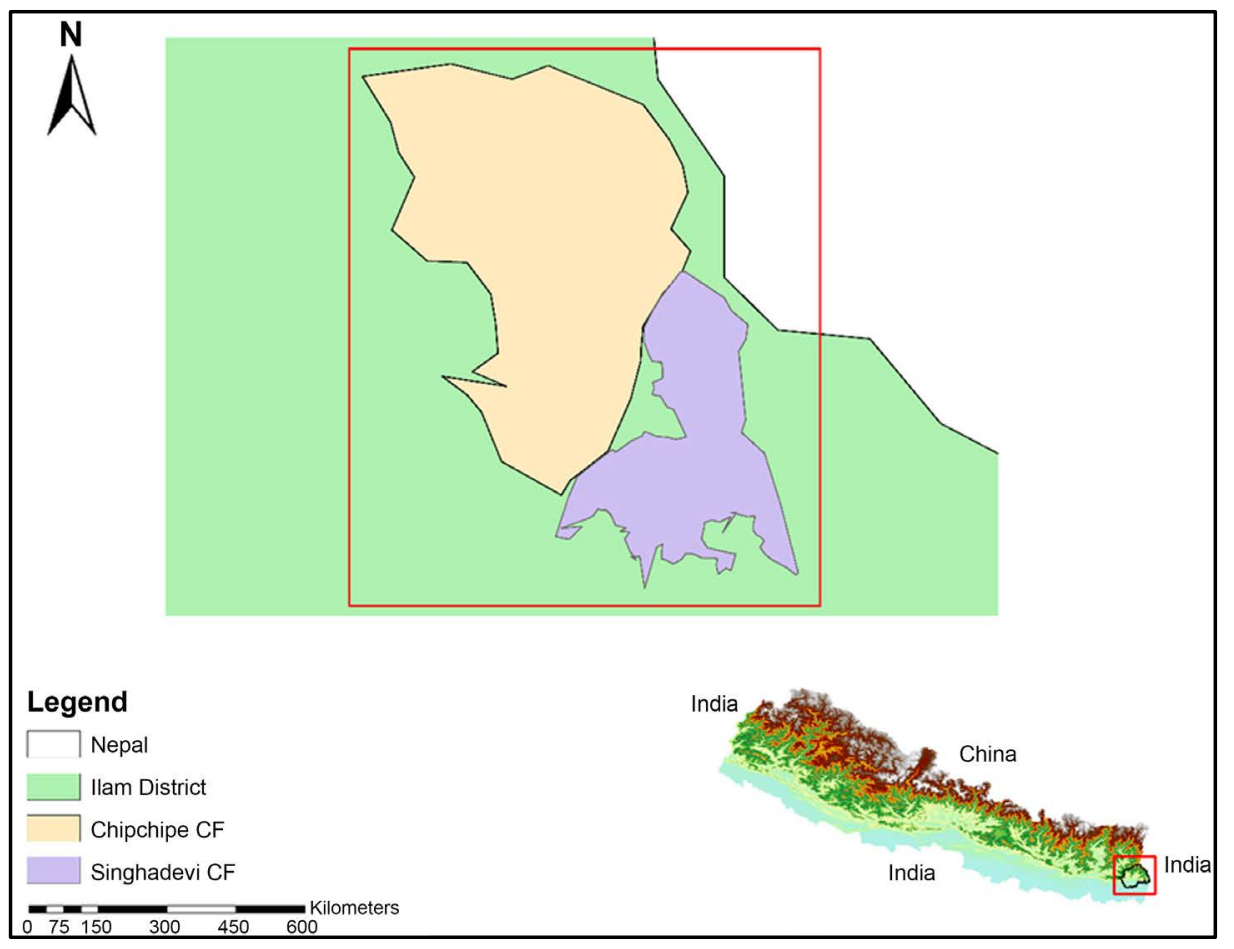

Figure 1. Study area.

community forests ranges from $2100 \mathrm{~m}$ to $2800 \mathrm{~m}$. The study area has a temperate broad-leaf ecosystem with high dominance of plant species like Rhododendron spp., Lithocarpus pachyphylla, Persea odoratissima, Dryopteris sparsa, and Arundinaria graminifolia [23].

The community forests are surrounded by two rural municipalities. Sherpas, the mountain-dwelling ethnic group are the subsistence farmers who practice conventional and unsustainable farming techniques and depend profoundly on the natural resources.

\section{Materials and Methods}

In total, 15 linear transects of an average length of $800 \mathrm{~m}$ were delineated in the study area (10 in Chipchipe CF and 5 in Singhadevi CF). Every linear transect was set at an altitudinal interval of approximately $100 \mathrm{~m}$ following the methodology provided by [24] and practiced by [8]. The linear transects in Singhadevi CF ranged from $2310 \mathrm{~m}$ to $2732 \mathrm{~m}$ whereas transects established in Chipchipe $\mathrm{CF}$ covered the elevation of $2318 \mathrm{~m}$ to $2754 \mathrm{~m}$ respectively. While traversing the linear transects, $10 \mathrm{~m} \times 10 \mathrm{~m}$ plots were outlined where livestock droppings and the indirect signs especially pellets of red panda were recorded. Besides, two plots were drawn in both initial and finishing points of every linear transect. Altogether, 58 plots were demarcated in the study area. Every plot in the linear transects was categorised according to the indirect signs. The plots containing only red panda evidences were marked as "red panda only plot" whereas plot containing livestock presence signs were noted as "livestock only plot". Addi- 
tionally, some plots were distinguished as habitat overlap plots where both red panda and livestock indirect marks were observed [17]. In some unexceptional cases, "non-sign" plots were also delineated predominantly in starting and terminating points of linear transects.

Geographic information such as latitudinal and longitudinal coordinates, elevation and slope was recorded from every plot by using the Global Positioning System (etrex 10). Estimated coverage percentage of different vegetation layers such as shrub layer, canopy and bamboo cover were noted from the surveyed plots. As red panda consume water quickly after eating, distance to nearest water source from the centre of plots was also measured [8] [25].

Altogether, 32 herders were interviewed regarding the socio-economic status, type and number of domesticated animals they rear, their involvement in red panda conservation actions, red panda importance and the possible hazards. Apart from, Key Informant Interviews (KII) was conducted where 4 forest guardians and 13 executive members of community forest user groups (CFUG) were surveyed to extract in-depth knowledge about the specific issues. Door to door survey was performed to obtain quality data from respondents.

In this study, tree species diversity of respective community forests was calculated by using Shannon-wiener diversity index $(H)=-\sum\left(n_{i} / N\right) * \log \left(n_{i} / N\right)$ where $n_{i}$ indicates the abundance of the $i$-th species of a given area whereas $N$ resembles total number of species present in the same area [26]. Jaccard's similarity index and Jaccard's distance was employed to measure the extent of resemblance and divergence in the habitats used by red panda and livestock.

Jaccard's similarity coefficient was determined by using the given formula:

$$
J=C / A+B-C[27][28]
$$

where, $A=$ number of plots showing red panda presence

$B=$ number of plots showing livestock presence

$C=$ number of plots showing both the presence of red panda and livestock

Likewise, Jaccard's distance was determined by subtracting the Jaccard similarity coefficient by $100 \%$ [29].

Multivariate analysis of variance (MANOVA) was conducted to understand the relationships between vegetation layers based on the interpretation of mean outcomes. Chi-square test ( $\mathrm{p}$ values) was conducted to evaluate the insight of local herders towards the effects of livestock grazing on red panda.

Microsoft Excel 365 and IBM SPSS Statistics 23.0 used for the data analysis. Besides, Google earth pro and ArcMap 10.3 were used to create the map of study area and extract geospatial information.

\section{Results}

\section{Habitat Availability and Preferences}

In Chipchipe CF, $48 \%$ plots were characterized as livestock only plot, $21 \%$ as red panda only plot, $18 \%$ as habitat overlap plot and $13 \%$ as non-sign plot $(N=$ 44). In Singhadevi CF, $71 \%$ were noted as livestock only plot and $29 \%$ as habitat 
overlap plot $(N=14)$

In total, 20 different tree species were recorded from the red panda potential habitat range in Chipchipe CF (Figure 2) suggesting Persea odoraissima, Lithocarpus pachyphylla, Rhodendrom falconeri, Schefflera impressa and Quercus spp as their favourable plant species.

Likewise, in Singhadevi CF, 11 different tree species were recorded from habitat overlap plots of livestock and red panda. Lithocarpus pachyphylla (24\%) had the highest abundance in red panda habitat range followed by Litsea elongata, Schefflera impressa and Rhododendron falconeri (Figure 3).

In Chipchipe CF, red panda only plot and habitat overlap plot presented a higher diversity index for tree species (Figure 4).

Habitat overlap plots of Singhadevi CF had higher tree diversity than in those plots which only contain livestock manure (Shannon-Wiener Diversity Index, $\mathrm{H}$ $=1.54$ ). The species richness calculated for different plots of Singhadevi CF had comparatively less diversity index value than Chipchipe CF.

Among 44 different plots demarcated in Chipchipe CF, bamboo abundance

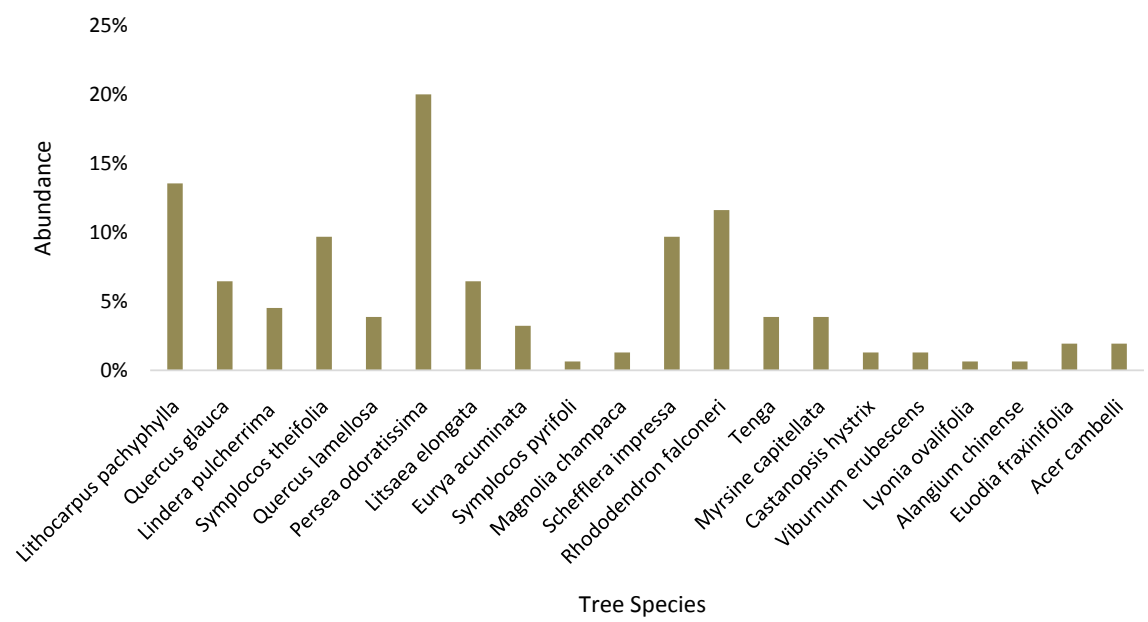

Figure 2. Dominance of tree species in red panda habitat in Chipchipe CF.

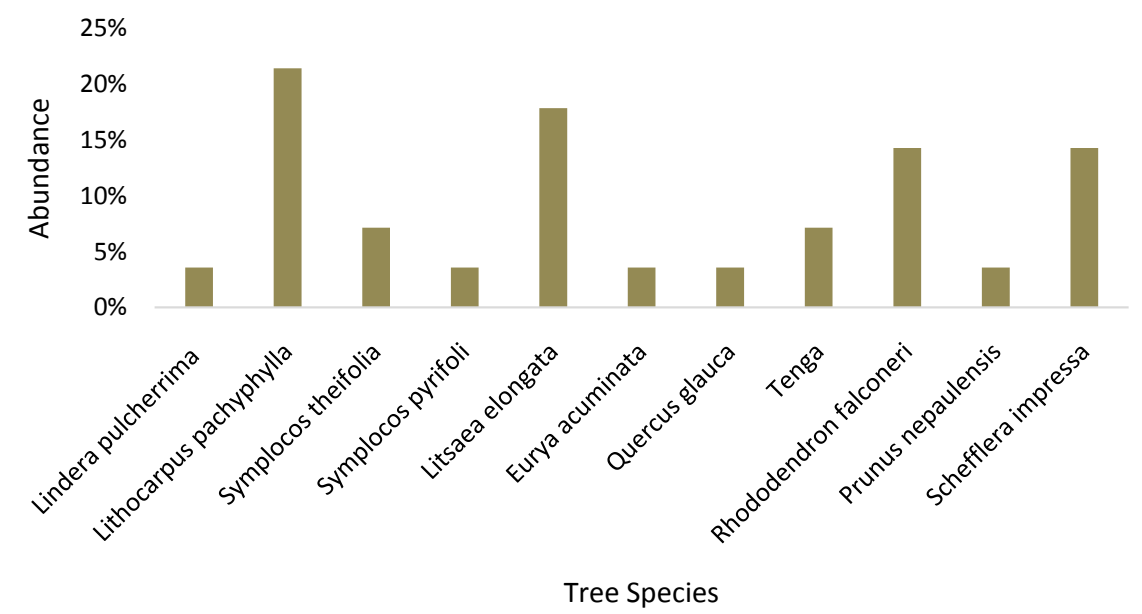

Figure 3. Dominance of tree species in red panda habitat in Singhadevi CF. 


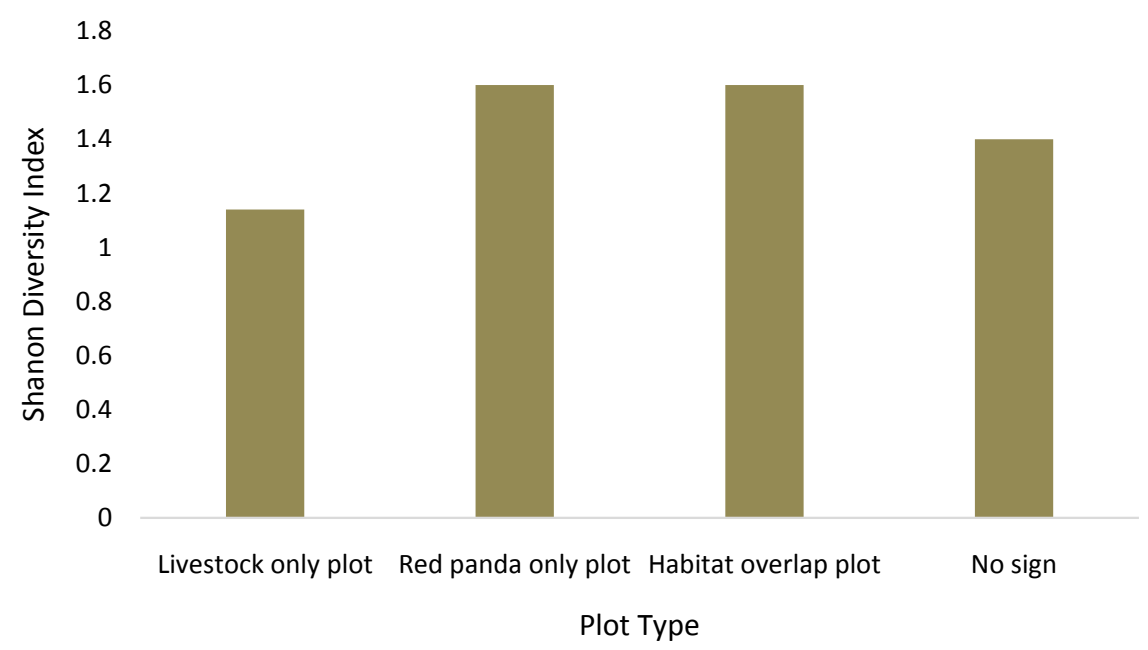

Figure 4. Shannon-Wiener Diversity Index as per the plot types in Chipchipe CF.

was the maximum where red panda pellets were solely discerned. Furthermore, canopy coverage in different plot types ranged from $40 \%-60 \%$, with red panda only plot to have the highest coverage. However, percentage coverage of the shrub layer was comparatively greater in those plots where only livestock droppings were marked (Table 1).

The statistical outcomes resulted from different multivariate tests (MANOVA) showed significance level lesser than 0.05 [i.e. $\mathrm{p}$-value $=0.047$ (Pillai's Trace), $\mathrm{p}$-value $=0.039$ (Wilks' Lambda), $\mathrm{p}$-value $=0.032$ (Hotelling's Trace), $\mathrm{p}$-value $=$ 0.02, (Roy's Largest Root). Vegetation layers coverage varies as per the plot type in the forest.

Specifically, in red panda only plot and habitat overlap plot/mutual plot of Chipchipe CF, faecal pellets groups were spotted within $160 \mathrm{~m}$ distance from the nearest water source (Figure 5). Grazing animals were found to prefer distance of $<425 \mathrm{~m}$ from the water source. Distance to the water source was the highest in non-sign plots i.e. $500 \mathrm{~m}$.

Canopy coverage was found widespread particularly in habitat overlap plots. Average shrub layer in livestock only plot of Singhadevi CF was higher. Lower abundance of bamboo species was detected from those plots which were shared both by red panda and livestock (Table 2). However, tree species richness was found significantly greater in habitat overlap plots as compared to livestock only plots. Likewise, red panda pellets in Singhadevi CF were also found near the water sources $(70 \mathrm{~m})$.

The probability value thus obtained from multivariate tests shows statistically significant outcome (i.e. $\mathrm{p}$-value $=0.046$ ).

\section{Habitat convergence between livestock and red panda}

There was a significant overlap in the resources shared by livestock and red panda. Livestock grazing was seen from $51 \%$ of red panda habitats in the study area [Jaccard's similarity index $(\mathrm{J})=0.51$, Jaccard distance $=0.49$ ] .

Potential red panda habitat determined from National Red Panda Survey 2016 
Table 1. Descriptive statistics for vegetation layers in different plots of Chipchipe CF.

\begin{tabular}{ccccc}
\hline \multicolumn{5}{c}{ Descriptive Statistics } \\
\hline \multirow{2}{*}{ Plot Type } & Mean & Std. Deviation & Number of plots \\
\hline \multirow{3}{*}{ Bamboo Abundance } & Livestock only plot & 411.67 & 403.82 & 21 \\
& Non-sign plot & 703.33 & 298.11 & 6 \\
& Red panda only plot & 852.22 & 491.73 & 9 \\
& Habitat overlap Plot & 812.5 & 281.26 & 8 \\
& Livestock only plot & 45.24 & 22.16 & 21 \\
& Non-sign plot & 42.5 & 11.73 & 6 \\
Canopy Coverage & Red panda only plot & 56.67 & 9.01 & 9 \\
& Habitat overlap Plot & 44.38 & 9.43 & 8 \\
& Livestock only plot & 23.91 & 21.89 & 21 \\
& Non-sign plot & 10 & 10.49 & 6 \\
Shrub Layer & Red panda only plot & 18.56 & 20.67 & 9 \\
& Habitat overlap Plot & 18.13 & 17.72 & 8
\end{tabular}

Table 2. Descriptive statistics for vegetation layers in different plots of Singhadevi CF.

\begin{tabular}{ccccc}
\hline \multicolumn{5}{c}{ Descriptive Statistics } \\
\hline \multirow{2}{*}{ Plot Type } & Mean & Std. Deviation & Number of plots \\
\hline \multirow{2}{*}{ Canopy Coverage } & Livestock only plot & 45.7 & 5.89 & 10 \\
& Habitat overlap plot & 48.75 & 6.75 & 4 \\
Shrub Layer & Livestock only plot & 35 & 10.36 & 10 \\
& Habitat overlap plot & 31.25 & 9.46 & 4 \\
Bamboo Abundance & Livestock only plot & 329 & 146.55 & 10 \\
& Habitat overlap plot & 250 & 91.29 & 4 \\
Shannon Diversity Index & Livestock only plot & 0.81 & 0.55 & 10 \\
& Habitat overlap plot & 1.54 & 0.2 & 4 \\
& Livestock only plot & 212 & 111.24 & 10 \\
\hline \multirow{2}{*}{ Distance to water source } & Habitat overlap plot & 70 & 37.19 & 4 \\
\hline
\end{tabular}

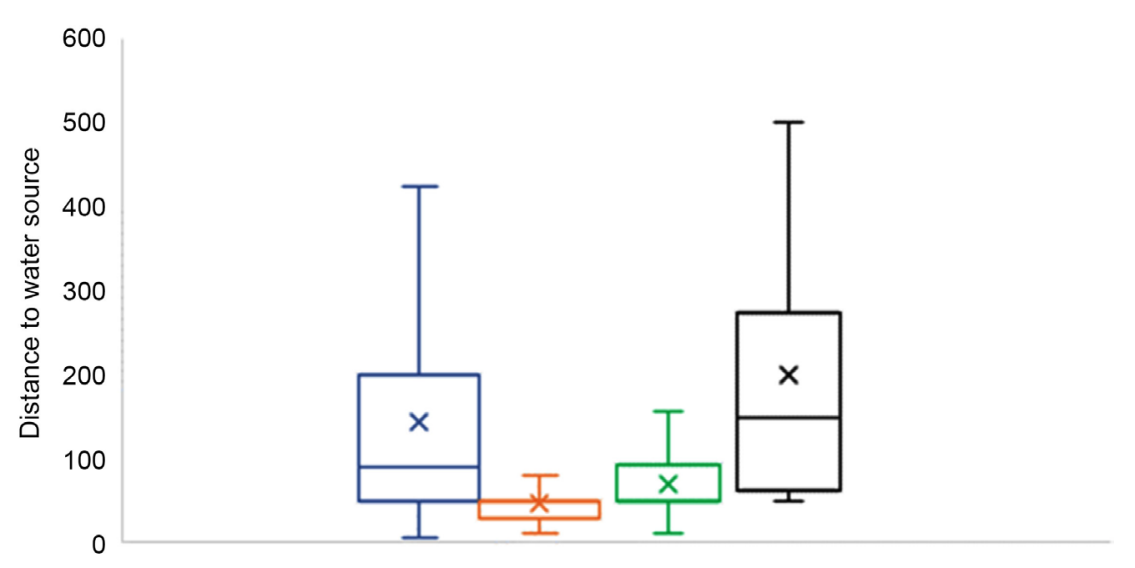

Plot Type

$\square$ Livestock only plot $\square$ Red Panda only plot $\square$ Mutual Plot $\square$ No sign

Figure 5. Distance from plot centre to water source in Chipchipe CF. 
gives us a basis to interpret red panda distribution and livestock interferences in the study area (Figure 6). 69.7\% of the surveyed plots which show livestock presence substantially overlaps with the potential habitat of red panda.

\section{Conservation Awareness Survey}

Livestock herding is a key source of income for these marginal communities. Approximately $37.5 \%$ of the herders rely only on livestock farming for their daily livelihoods whilst remaining $62.5 \%$ of their population's subsidiary income source includes agricultural production and business.

The income chart illustrates the gross annual earning of livestock herders. $12.5 \%$ of livestock herders earn 20,000 NPR (Nepalese Rupee) per annum whereas $37.5 \%$ of herder's income is estimated to range from 20,000 - 40,000 NPR per annum (Figure 7). Remaining, 50\% of the livestock herders acquire 40,000 60,000 NPR and above 60,000 NPR by selling livestock commodities.
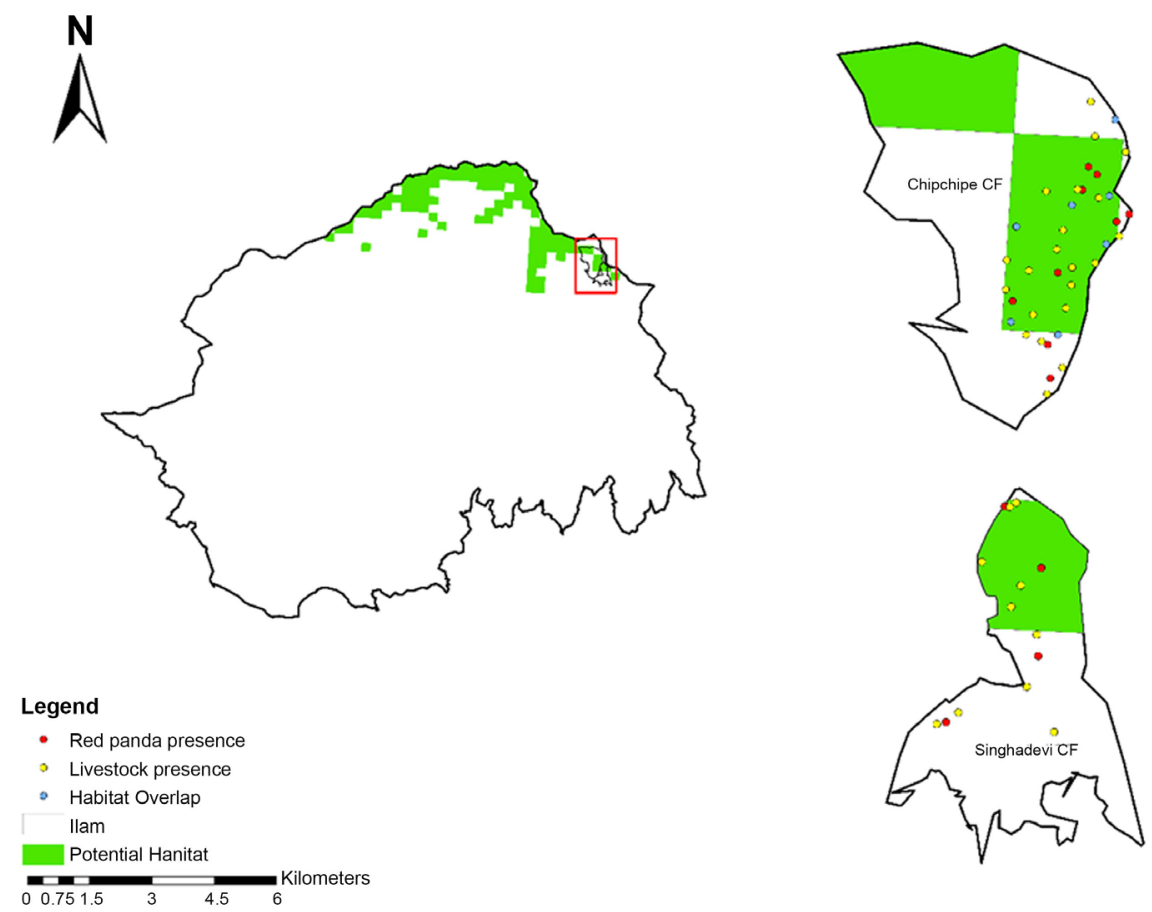

Figure 6. Habitat overlap between red panda and grazing animals in the study area.

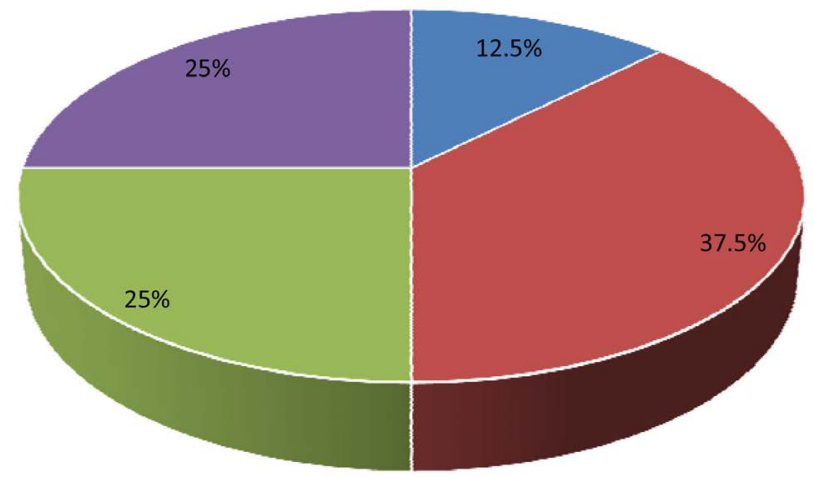

NPR

- 20000

|- 20000-40000

= 40000-60000

- $>60000$

Figure 7. Annual Income from livestock farming. 
Mainly, there are three types of livestock that grazes in the community forest. Cow was the highest in number among the livestock followed by ox and horse. During the study period, 512 livestock were found grazing within the red panda habitat. In total, $87.5 \%$ of herders are unaware of the conservation importance of red panda. However, $12.5 \%$ of herders are enlightened about the significance of such endangered species. In total, $75 \%$ of the local herders are vigilant about the fact that livestock grazing could have negative threat to the survival of red pandas. Overall, $12.5 \%$ of herder's believe that grazing animals do not possess threat to red pandas whilst the rest of the herders are uncertain about the consequences. The significance level obtained from the chi-square test is less than 0.05 i.e. $\mathrm{p}$-value $=0.04$ KII alleviated in understanding the knowledge gaps aroused from questionnaire survey. Every key informant interviewee believed that extensive grazing practices within the forest is causing negative effect on the natural resources and wildlife habitat.

\section{Discussion}

Red panda mostly prefers Persea odoraissima, Lithocarpus pachyphylla, Schefflera impressa, Rhodendrom falconeri, Quercus spp. and Litsea elongata. A corresponding study carried out in Jamuna and Mabu Village Development Committee of Ilam district showed Schefflera impressa as one of the most-favoured tree species by red panda [7]. However, [17] detected contradictory results for a similar type of study where red panda preferred tree species like Betula Utilis and Abies spectabilis and Acer spp. The reason behind disparate findings is that the study area (Dhorpatan Hunting Reserve) lies in Western Nepal, which has comparatively different topographical features and forest ecosystem. The diversity indices for the plots comprising red panda signs varied from 1.54 to 1.60 . The value range shows moderate plant richness in red panda habitat. Kong et al. [30] found similar increment in the vegetation diversity of wild red panda habitat (Shannon Index, $H=1.3$ to 2.9 ).

Bamboo presence in $93 \%$ of red panda signs plot portrays about the significance of bamboo as one of the key factors affecting their distribution. Similarly, in Jigme Singye Wangchuk National Park, red panda scats were mostly detected in those areas where bamboo species like Arundinaria racemose and Borindina grossa were abundant [31]. Panthi et al. [32] explored the nutritional composition of red panda pellets and found Arundinaria spp. as frequently consumed plant species. However, in contrast to red panda only plots, bamboo abundance was found to be minimal in habitat overlap plots of both community forests. This might be due to livestock grazing and the trampling effect that might had caused die-off of bamboo species. Grazing practices decrease bamboo accessibility and prevent it to grow at adequate height requisite for red pandas to forage [6] [33]

Red panda presence signs were detected from the sites with denser canopy cover (44\% - 57\%). Williams [7] quantified that red panda prefers forests with 
more than $30 \%$ of canopy cover. Additionally, red panda might use canopy cover as their defence mechanism to circumvent their predators. However, canopy coverage had less influence on grazing animals. The shrub layer was found comparatively higher in livestock plots of both community forests. The reason behind the larger occupancy of the shrub layer probably signifies the positive response of livestock towards the available shrubs in the surveyed plots. Red panda pellets in the community forests were present at a minimal distance (100 $\mathrm{m}$ ) from the water sources. Water source accessibility is one of the main factors that determine habitat utilization either by wildlife species or grazing animals [12]. An analogous study conducted in Chitwan-Annapurna Landscape of Nepal demonstrated the preferable distance of $100 \mathrm{~m}-200 \mathrm{~m}$ [8].

Red panda habitat in the study area shows significant overlapping with livestock presence. This might be because herders, livestock and red panda prefer habitat nearer to water sources. Similarly, Panthi et al. [17] observed livestock grazing activities in 53\% of red panda habitat range in Dhorpatan Hunting reserve. Livestock faeces exhibited a positive correlation with red panda presence in the Central Himalayas [18]. The outcomes depicted that such high grazing intensity could threaten the habitat and long-term survival of red panda. Schieltz and Rubenstein, [34] found that the species such as red panda which requires dense canopy cover are adversely affected by grazing as compared to the species acclimatizing to open environments. Through much of its spatial scale, livestock grazing has been posing serious threat either by inhabiting their suitable habitat or competing for same food resources [16]. Also, livestock has been recognized as a potential source for the transmission of zoonotic parasites [8]. Lama et al. [35] found that parasites such as Trichuris spp reported from livestock is the underlying cause of bloody colitis, anaemia and sometimes even death in red pandas. Observed rates of livestock use in the study area clearly portrays about the profound impacts on red panda habitat. Acharya et al. [18] also concluded that the non-protected areas such as community forests showed higher abundance of livestock droppings than protected areas (PA) as there are some restrictions of carrying out grazing within core PAs habitat.

Though, livestock herding is considered as a principal economic occupation of these indigenous people, the total revenue attainable is inadequate. The significance level obtained from the conservation awareness survey demonstrates that livestock grazing possesses impacts on red pandas. Furthermore, the key informant interviewees claimed that rivalry for the same food, depletion of vegetation cover and degradation of forest are the main challenges to red panda provoked by grazing pressures.

\section{Conclusions}

The research provides a vivid overview of grazing impacts on red panda. Unsustainable grazing has negatively impacted the occurrence and habitat of red panda in the study zone. The absence of effective strategies related to livestock 
herding management has resulted in haphazard grazing inside the community forest area. The habitat of red panda within the research area is disturbed due to unregulated grazing activities. If livestock use cannot be moderated, grazing should be carried out outside the red panda core habitat. Potential habitat of red panda should be assigned as a buffer zone in order to minimize the illegal use of resources within the community forests. Local herders should be well trained about improved herding practices such as stall feeding, set stocking and rotational grazing. Additionally, the formulation of discrete national policy for livestock management would significantly help in the minimization of arising problems. The resulted outcomes could provide baseline information to the researchers for carrying out rigorous studies hereafter.

Both community forests extend over low altitudinal mountainous scale resembling the similar type of ecosystem. The length of transects was not uniform due to the sloped terrain and this might not precisely generalize the vegetation abundance pattern.

\section{Acknowledgements}

We are thankful to Red Panda Network and Small Mammals Conservation Research Foundation for providing financial assistance to conduct this thesis research. We sincerely acknowledge the support of the CFUGs who contributed directly or indirectly in the field.

\section{Conflicts of Interest}

The authors declare no conflicts of interest regarding the publication of this paper.

\section{References}

[1] Smith, A.B. and Santos, M.J. (2019) Testing the Ability of Species Distribution Models to Infer Variable Importance. https://doi.org/10.1101/715904

[2] Thapa, A., Hu, Y., Aryal, P.C., Singh, P.B., Shah, K.B. and Wei, F. (2020) The Endangered Red Panda in Himalayas: Potential Distribution and Ecological Habitat Associates. Global Ecology and Conservation, 21, e00890.

https://doi.org/10.1016/j.gecco.2019.e00890

[3] Kandel, K., Huettmann, F., Suwal, M.K., Regmi, G.R., Nijman, V., Nekaris, K.A.I., Lama, S.T., Thapa, A., Sharma, H.P. and Subedi, T.R. (2015) Rapid Multi-Nation Distribution Assessment of a Charismatic Conservation Species Using Open Access Ensemble Model GIS Predictions: Red Panda (Ailurus fulgens) in the Hindu-Kush Himalaya Region. Biological Conservation, 181, 150-161. https://doi.org/10.1016/j.biocon.2014.10.007

[4] Titeux, N., Aizpurua, O., Hollander, F.A., Sardà-Palomera, F., Hermoso, V., Paquet, J.Y., Mestdagh, X., Settele, J., Brotons, L. and Van Dyck, H. (2019) Ecological Traps and Species Distribution Models: A Challenge for Prioritizing Areas of Conservation Importance. Ecography, 43, 365-375. https://doi.org/10.1111/ecog.04783

[5] Pradhan, S., Saha, G.K. and Khan, J.A. (2001) Ecology of the Red Panda Ailurus fulgens in the Singhalila National Park, Darjeeling, India. Biological Conservation, 
98, 11-18. https://doi.org/10.1016/S0006-3207(00)00079-3

[6] Yonzon, P.B. and Hunter Jr., M.L. (1991) Cheese, Tourists, and Red Pandas in the Nepal Himalayas. Conservation Biology, 5, 196-202. https://doi.org/10.1111/j.1523-1739.1991.tb00124.x

[7] Williams, B.H. (2004) Red Panda in Eastern Nepal: How Do They Fit into Ecoregional Conservation of the Eastern Himalaya. Conservation Biology in Asia, 16, 236-250.

[8] Bista, D., Shrestha, S., Kunwar, A.J., Acharya, S., Jnawali, S.R. and Acharya, K.P. (2017) Status of Gastrointestinal Parasites in Red Panda of Nepal. PeerJ, 5, e3767. https://doi.org/10.7717/peerj.3767

[9] Glatston, A., Wei, F., Zaw, T. and Sherpa, A. (2015) Ailurus fulgens (Errata Version Published in 2017). The IUCN Red List of Threatened Species 2015: e.T714A110023718. https://doi.org/10.2305/IUCN.UK.2015-4.RLTS.T714A45195924.en

[10] Jnawali, S., Leus K., Molur, S., Glatston, A. and Walker, S. (2012) Red Panda (Ailurus fulgens). Population and Habitat Viability Assessment (PHVA) and Species Conservation Strategy (SCS) Workshop Report. National Trust for Nature Conservation, Kathmandu, Nepal, Conservation Breeding Specialist Group and Zoo Outreach Organization, Coimbatore.

[11] Panthi, S., Khanal, G., Acharya, K.P., Aryal, A. and Srivathsa, A. (2017) Large Anthropogenic Impacts on a Charismatic Small Carnivore: Insights from Distribution Surveys of Red Panda Ailurus fulgens in Nepal. PLoS ONE, 12, e0180978. https://doi.org/10.1371/journal.pone.0180978

[12] Bhatta, M., Shah, K.B., Devkota, B., Paudel, R. and Panthi, S. (2014) Distribution and Habitat Preference of Red Panda (Ailurus fulgens fulgens) in Jumla District, Nepal. Open Journal of Ecology, 4, 989. https://doi.org/10.4236/oje.2014.415082

[13] Yonzon, P.B., Chaudhary, C. and Vaidya, B. (1997) Status of the Red Panda in the Himalaya. A Resources Nepal. Kathmandu and Metropolitan Toronto Zoo Project, Toronto, 21.

[14] Jnawali, S. (2011) The Status of Nepal's Mammals: The National Red List Series. Department of National Parks and Wildlife Conservation, Kathmandu.

[15] Bista, D., Paudel, P.K., Jnawali, S.R., Sherpa, A.P., Shrestha, S. and Acharya, K.P. (2019) Red Panda Fine-Scale Habitat Selection along a Central Himalayan Longitudinal Gradient. Ecology and Evolution, 9, 5260-5269. https://doi.org/10.1002/ece3.5116

[16] Hull, V., Zhang, J., Zhou, S., Huang, J., Vina, A., Liu, W., Tuanmu, M.N., Li, R., Liu, D., Xu, W. and Huang, Y. (2014) Impact of Livestock on Giant Pandas and Their Habitat. Journal for Nature Conservation, 22, 256-264. https://doi.org/10.1016/j.jnc.2014.02.003

[17] Panthi, S., Aryal, A., Raubenheimer, D., Lord, J. and Adhikari, B. (2012) Summer Diet and Distribution of the Red Panda (Ailurus fulgens fulgens) in Dhorpatan Hunting Reserve, Nepal. Zoological Studies, 51, 701-709.

[18] Acharya, K.P., Shrestha, S., Paudel, P.K., Sherpa, A.P., Jnawali, S.R., Acharya, S. and Bista, D. (2018) Pervasive Human Disturbance on Habitats of Endangered Red Panda Ailurus fulgens in the Central Himalaya. Global Ecology and Conservation, 15, e00420. https://doi.org/10.1016/j.gecco.2018.e00420

[19] Sharma, H.P., Swenson, J. and Belant, J.L. (2014) Seasonal Food Habits of the Red Panda (Ailurus fulgens) in Rara National Park, Nepal. Hystrix, 25, 47-50.

[20] Bartolome, J.W., Allen-Diaz, B.H., Barry, S., Ford, L.D., Hammond, M., Hopkinson, 
P., Ratcliff, F., Spiegal, S. and White, M.D. (2014) Grazing for Biodiversity in Californian Mediterranean Grasslands. Rangelands, 36, 36-43. https://doi.org/10.2111/Rangelands-D-14-00024.1

[21] Barry, S., Bush, L., Larson, S. and Ford, L.D. (2015) The Benefits of Grazing-Livestock Grazing: A Conservation Tool on California's Annual Grasslands. Division of Agriculture and Natural Resources, Richmond. https://doi.org/10.3733/ucanr.8517

[22] Red Panda Network (RPN) (2017) PIT Red Panda Protected Forest [Online]. https://www.redpandanetwork.org/pit-red-panda-protected-forest

[23] Bhattarai, K.R. (2016) Documentation of Flora of Ilam, East Nepal. District Plant Resources Office, Ilam.

[24] Ministry of Forests and Soil Conservation (2015) Red Panda Field Survey and Protocol for Community Based Monitoring. Ministry of Forests and Soil Conservation, Singha Durbar.

[25] Yonzon, P.B. (1989) Ecology and Conservation of the Red Panda in the Nepal Himalayas. University of Maine, Orono.

[26] Mårtensson, R. (2016) Species and Biological Diversity-Choices of Diversity Indices and Their Potential Consequences for Nature Conservation [Online].

http://lup.lub.lu.se/luur/download?func=downloadFile\&recordOId=8876148\&fileO $\underline{\mathrm{Id}=8876149}$

[27] Real, R. and Vargas, J.M. (1996) The Probabilistic Basis of Jaccard's Index of Similarity. Systematic Biology, 45, 380-385. https://doi.org/10.1093/sysbio/45.3.380

[28] Real, R. (1999) Tables of Significant Values of Jaccard's Index of Similarity. Miscellania Zoologica, 22, 29-40.

[29] Stephanie (2016) Jaccard Index/Similarity Coefficient [Online]. https://www.statisticshowto.datasciencecentral.com/jaccard-index

[30] Kong, F., Zhao, J., Han, S., Zeng, B., Yang, J., Si, X., Yang, B., Yang, M., Xu, H. and Li, Y. (2014) Characterization of the Gut Microbiota in the Red Panda (Ailurus fulgens). PLoS ONE, 9, e87885. https://doi.org/10.1371/journal.pone.0087885

[31] The Rufford Foundation (2019) Habitat Status and Conservation Threats of the Red Panda in Jigme Singye Wangchuck National Park [Online]. https://www.rufford.org/projects/kesang_wangchuk

[32] Panthi, S., Coogan, S.C., Aryal, A. and Raubenheimer, D. (2015) Diet and Nutrient Balance of Red Panda in Nepal. The Science of Nature, 102, 54. https://doi.org/10.1007/s00114-015-1307-2

[33] Sharma, H.P., Belant, J.L. and Swenson, J.E. (2014) Effects of Livestock on Occurrence of the Vulnerable Red Panda Ailurus fulgens in Rara National Park, Nepal. Oryx, 48, 228-231. https://doi.org/10.1017/S0030605313001403

[34] Schieltz, J.M. and Rubenstein, D.I. (2016) Evidence Based Review: Positive versus Negative Effects of Livestock Grazing on Wildlife. What Do We Really Know? Environmental Research Letters, 11, Article ID: 113003. https://doi.org/10.1088/1748-9326/11/11/113003

[35] Lama, S.T., Lama, R.P., Regmi, G.R. and Ghimire, T.R. (2015) Prevalence of Intestinal Parasitic Infections in Free-Ranging Red Panda Ailurus fulgens Cuvier, 1825 (Mammalia: Carnivora: Ailuridae) in Nepal. Journal of Threatened Taxa, 7, 7460-7464. https://doi.org/10.11609/JoTT.o4208.7460-4 


\section{Abbreviations and Acronyms}

CF: Community Forest

KII: Key Informant Interview

MANOVA: Multivariate analysis of variance

NTNC: National Trust for Nature Conservation

PA: Protected Areas

PHVA: Population and Habitat Viability Assessment

CFUG: Community Forest User Groups 